\title{
A saúde na Agenda 2030 de Desenvolvimento Sustentável na América Latina: quem está publicando?
}

\author{
Health in the Sustainable Development Agenda 2030 in Latin America: \\ who is publishing?
}

\section{La salud en la Agenda 2030 de Desarrollo Sostenible en América Latina: ¿Quién está publicando?}

Cristiane Machado Quental ${ }^{1, a}$

cristiane.quental@fiocruz.br | https://orcid.org/0000-0002-6907-3684

\author{
Miryam Souza Minayo ${ }^{1, b}$ \\ miryam.minayo@fiocruz.br | https://orcid.org/0000-0003-3521-3113 \\ Danúzia Rocha Paula ${ }^{2, c}$ \\ rochadanuzia@gmail.com | https://orcid.org/ooo0-0001-8966-4839
}

\author{
Rodrigo Teixeira Amâncio ${ }^{3, d}$ \\ amancio.rt@gmail.com | https://orcid.org/0000-0001-5155-7536 \\ Luiz Augusto Galvão ${ }^{1, e}$ \\ luiz.galvao@fiocruz.br | https://orcid.org/0000-0002-3918-0286
}

Fernando Augusto Bozza ${ }^{3, d}$

bozza.fernando@gmail.com | https://orcid.org/0000-0003-4878-0256

Paulo Marchiori Buss ${ }^{1 . f}$

buss@fiocruz.br | https://orcid.org/0000-0002-9944-9195

\footnotetext{
${ }^{1}$ Fundação Oswaldo Cruz, Presidência, Centro de Relações Internacionais em Saúde. Rio de Janeiro, RJ, Brasil.

2 Universidade Federal Fluminense, Biblioteca da Faculdade de Medicina. Niterói, RJ. Brasil.

${ }^{3}$ Fundação Oswaldo Cruz, Instituto Nacional de Infectologia Evandro Chagas. Rio de Janeiro, RJ, Brasil.

a Doutorado em Administração pela Universidade Federal do Rio de Janeiro.

b Doutorado em Direito Internacional e Relações Internacionais pela Universidad Complutense de Madrid.

c Mestrado em Ciência da Informação pela Universidade Federal Fluminense.

d Doutorado em Biologia Celular e Molecular pela Fundação Oswaldo Cruz.

e Doutorado em Saúde Coletiva pela Universidade Federal do Rio de Janeiro.

f Mestrado em Medicina Social pela Universidade do Estado do Rio de Janeiro.
}

\section{Resumo}

Este artigo apresenta um trabalho que consistiu no levantamento quantitativo das publicações sobre a Agenda 2030 de Desenvolvimento Sustentável na América Latina, ou que foram escritas por autores afiliados às instituições da região, focando no tema saúde. Foram realizadas buscas em bancos de dados, para o período entre janeiro de 2009 e maio de 2017, e encontradas 127 publicações voltadas para o Objetivo de Desenvolvimento Sustentável 3 - Boa Saúde e Bem-Estar. A maior parcela dos resultados (92\%) é constituída de artigos científicos que foram descritos segundo o ano e veículo de publicação, instituições de filiação dos autores e sua localização. Quase a metade das publicações (47\%) foi feita sem a participação de organizações latino-americanas e em apenas 30\% dos casos o primeiro autor pertence a uma organização latino-americana. Esse quadro se torna mais nítido quando se observa a concentração da produção latinoamericana em torno de organizações brasileiras: a produção científica sobre as resoluções da Agenda 2030 em relação à saúde ainda está ausente em grande parte do território latino-americano.

Palavras-chave: Agenda 2030; Objetivos do Desenvolvimento Sustentável; Desenvolvimento sustentável; Agenda pós-2015; América Latina. 


\begin{abstract}
This article presents a work that consisted of a quantitative research about publications on Agenda 2030 on Sustainable Development in Latin America, or written by authors affiliated with institutions in the region, focusing on health. A search using databases on the subject for the period between January 2009 and May 2017 was made, and 127 publications focusing on Sustainable Development Goal 3 - Good Health and Well-Being. The majority (92\%) of the publications were scientific articles and were described in relation to the year and vehicle of publication, authors' affiliation institutions and their location. Almost half of the publications (47\%) were made without the participation of Latin American organizations and only 30\% of cases have the first author who belongs to a Latin American organization. This picture becomes clearer when we see the concentration of Latin American production at Brazilian organizations: scientific production on Agenda 2030 regarding health is still absent in much of the Latin American territory.
\end{abstract}

Keywords: 2030 Agenda; Sustainable Development Goals; Sustainable development; Post-2015 Development Agenda; Latin America.

\title{
Resumen
}

Este artículo presenta un trabajo que ha consistido en el levantamiento cuantitativo de las publicaciones sobre la Agenda 2030 en América Latina o que fueron escritas por autores afiliados a instituciones de la región con el foco en salud. Se realizaron búsquedas en bancos de datos para el período entre 2009 y mayo de 2017. Se encontraron 127 publicaciones sobre el Objetivos de desarrollo sostenible 3 - Salud y Bienestar. Constituyen la mayoría (92\%) artículos científicos que fueron descritos según el año y el vehículo de publicación, las instituciones de filiación de los autores y su ubicación. En casi la mitad de las publicaciones (47\%) no hubo participación de organizaciones latinoamericanas y solamente en 30\% de los casos el primer autor pertenece a una organización latinoamericana. Este cuadro es más nítido cuando observada la concentración de la producción latinoamericana en organizaciones brasileñas: La producción científica sobrela Agenda 2030 en el área de la salud todavía está ausente en gran parte de la América Latina.

Palabras clave: Agenda 2030; Objetivos del Desarrollo Sostenible; Desarrollo sostenible; Agenda Pos2015; América Latina.

\footnotetext{
Contribuição dos autores:

Cristiane Quental, Miryam Minayo trabalharam em todas as etapas de elaboração e redação do artigo.

Danúzia da Rocha de Paula colaborou no levantamento bibliográfico.

Rodrigo Teixeira Amâncio, Luis Augusto Galvão, Fernando Augusto Bozza e Paulo Buss contribuíram na redação do artigo.
}

Declaração de conflito de interesses: não há.

Fontes de financiamento: International Research Development Center (Canadá).

Considerações éticas: não há

Agradecimentos/Contribuições adicionais: este trabalho foi conduzido no marco do projeto Policy Research Institutions and the Health SDGs: Scoping SDG Governance Arrangements across Latin America.

Histórico do artigo: submetido: 28 maio. 2019 | aceito: 04 set. 2019 | publicado: 20 dez. 2019.

Apresentação anterior: Este artigo é resultado de um desdobramento do projeto Policy Research Institutions and the Health SDGs: Scoping SDG Governance Arrangements across Latin America.

Licença CC BY-NC atribuição não comercial. Com essa licença é permitido acessar, baixar (download), copiar, imprimir, compartilhar, reutilizar e distribuir os artigos, desde que para uso não comercial e com a citação da fonte, conferindo os devidos créditos de autoria e menção à Reciis. Nesses casos, nenhuma permissão é necessária por parte dos autores ou dos editores. 


\section{Introdução}

A Agenda 2030 é um plano de ação para o desenvolvimento sustentável do planeta, adotado pelos chefes de Estado e de Governo dos 193 países membros da Organização das Nações Unidas (ONU) no final de 2015, que deverá orientar as políticas nacionais e ações de cooperação internacional pelos próximos 15 anos. A proposta se construiu sobre o legado dos Objetivos de Desenvolvimento do Milênio (ODM), da Declaração do Rio sobre Meio Ambiente e Desenvolvimento (Rio+20) e com base em consultas públicas e no envolvimento da sociedade civil e demais partes interessadas em todo o mundo.

O desenvolvimento sustentável é entendido nas suas três dimensões: econômica, social e ambiental. Busca-se, então, o bem-estar das pessoas e sua prosperidade, com crescimento sustentado em harmonia com a proteção ao planeta, para que suporte as necessidades das gerações atuais e futuras, e em paz.

O plano descrito no documento Transformar nosso Mundo: a Agenda 2030 para o Desenvolvimento Sustentável ${ }^{1}$ é considerado ambicioso. Ele estabelece 17 objetivos (e 169 metas) inter-relacionados e interdependentes, apresentados na Figura 1, que exigem soluções integradas. Esses objetivos tratam de temáticas diversas que incluem questões centrais como erradicação da pobreza, segurança alimentar e agricultura, saúde e bem-estar, educação de qualidade, igualdade de gênero, redução das desigualdades, energia, água e saneamento, condições decentes de trabalho, padrões sustentáveis de produção e de consumo, mudanças climáticas, cidades sustentáveis, proteção e uso sustentável dos oceanos e dos ecossistemas terrestres, crescimento econômico inclusivo, paz, justiça e meios de implementação desses valores.
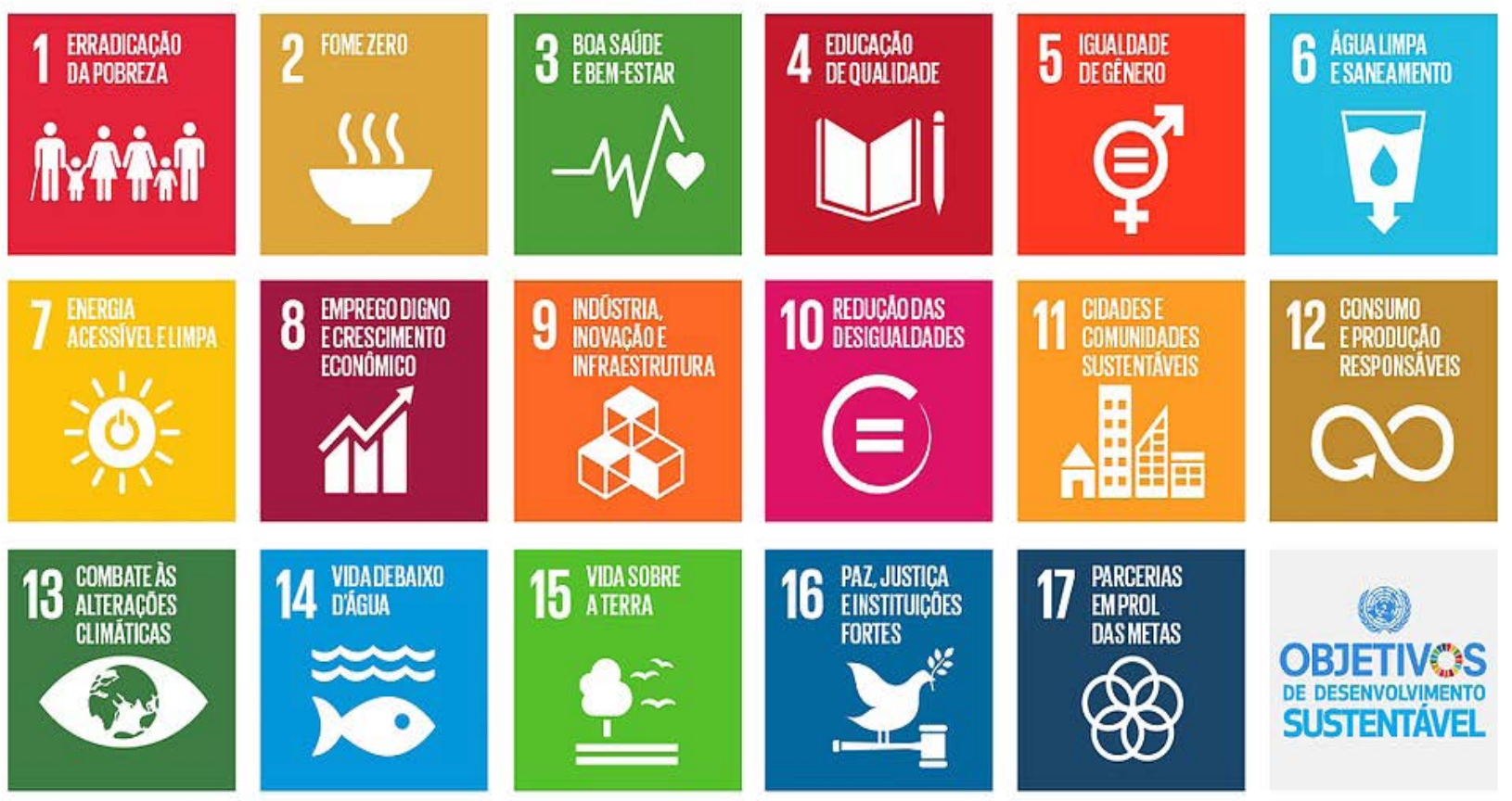

Figura 1 - Objetivos de Desenvolvimento Sustentável (ODS) - Agenda 2030 Fonte: ONU Brasil, 20182

Cada país deve adaptar esses objetivos e metas universais às realidades e prioridades nacionais, exercendo livremente sua soberania. Desse modo, numerosos países em todo o planeta estão montando suas estruturas de governança para a implementação da Agenda.

Na América Latina, os governos lideram o processo, mas a participação da sociedade civil tem sido, em geral, tímida ${ }^{3}$, limitando a implementação dos Objetivos de Desenvolvimento Sustentável (ODS) na região.

No estudo aqui apresentado, parte-se do pressuposto que as redes de instituições acadêmicas e os thinktanks (policy research organizations) ${ }^{4}$, quando articulados, podem contribuir para a aceleração da 
implementação da Agenda na América Latina, ao subsidiarem a implementação dos ODS nacionalmente. O trabalho teve como objetivo identificar as instituições acadêmicas e thinktanks atuantes no tema da implementação da Agenda 2030 e de seus ODS na América Latina, com foco na saúde. Neste artigo, apresentam-se os resultados do mapeamento realizado.

\section{Metodologia}

Optou-se por identificar as instituições e thinktanks indiretamente, por meio de sua produção bibliográfica publicada. Assim, realizou-se uma busca sistemática dessas publicações em repositórios e bancos de dados eletrônicos. Pesquisou-se a quantidade e a distribuição da produção científica por temas, veículos de publicação, instituições de afiliação dos autores e países onde se localizam. A metodologia de trabalho é detalhada a seguir.

\section{Estratégia de busca}

Para realização deste levantamento bibliográfico foram utilizadas as seguintes palavras-chave:

- em inglês - 'sustainable development goals' OR ‘2030 agenda' OR ‘2030 agenda for sustainable development' OR 'post-2015 Agenda' OR 'post 2015 agenda' OR 'post-2015 development agenda'

- em português - 'objetivos de desenvolvimento sustentável' OR ‘Agenda 2030’ OR 'Agenda 2030 para desenvolvimento sustentável' OR ‘Agenda pós-2015' OR ‘Agenda de desenvolvimento pós-2015’

- em espanhol - 'objetivos de Desarrollo Sostenible' OR ‘Agenda 2030’ OR ‘Agenda 2030 para el desarrollo Sostenible' OR ‘Agenda Post-2015’ OR ‘Agenda post-2015 para el desarrollo’

Utilizou-se como recorte local 'América Latina' e seus países ('Mexico' OR 'Argentina' OR ‘Bolivia' OR 'Brasil' OR ‘Chile' OR ‘Colombia' OR ‘Costa Rica' OR ‘Cuba' OR ‘Equador' OR 'El Salvador' OR ‘Guatelama' OR ‘Haiti' OR ‘Honduras' OR 'Nicaragua' OR 'Panama' OR 'Paraguai' OR 'Peru' OR 'República Dominicana' OR 'Uruguai' OR 'Venezuela'), também com a variação de grafia dos três idiomas. Como recorte temporal utilizou-se o período de janeiro de 2009 a maio de 2017.

\section{Fontes de informação}

Foram utilizadas bases de dados bibliográficos gerais, como a Web of Science e a Scopus, bases voltadas para a área da saúde, como a Biblioteca Virtual em Saúde e a Pubmed/Medline e bases de dados que pudessem trazer referências específicas sobre e sustentabilidade e saúde, essas últimas a partir da busca realizada por Galvão ${ }^{5}$. Essas bases estão listadas a seguir.

- Bases de dados bibliográficos: Portal Regional da Biblioteca Virtual de Saúde (BVS); Scopus; Web of Science; CINAHL-Nursing \& Allied Health Literature; Science Direct; AGRICOLA; Food Science and Technology Abstracts (FSTA); Scientific Eletronic Library (SciELO); Pubmed/ Medline; Sociological Abstracts; $\underline{\text { ASSIA }}$; GreenFILE; $\underline{\text { AGRIS; }}$ Biblioteca Digital Brasileira de Teses e Dissertações (BDTD); Open Access Theses and Dissertations (OATD); Networked Digital Library of Theses and Dissertations (NDLTD).

- Bases de revisão sistemática: Cochrane Library Plus; Campbell Library; EPPI Centre; HealthEvidence Canada; National Institute for Health and Care Excellence; The Community Guide; International Initiative for Impact Evaluation; Institute Evidence-check Library; Paediatric Economic Database Evaluation; Economic Literature (EconLit); NHS Economic Evaluation Database (NHSEED).

- Outras fontes de informação: Google acadêmico. 


\section{Extração de dados}

A busca realizada nas bases de dados resultou em 278 referências que atendiam a uma das duas condições abaixo:

- autor vinculado a uma organização latino-americana

- autor não vinculado a uma organização latino-americana, mas que trata especificamente da Agenda 2030 e de seus ODS na América Latina

Essas publicações foram descritas de acordo com as seguintes variáveis:

1. Título do documento;

2. Autores;

3. Filiação dos autores: nome da organização à qual o autor está filiado e país onde está localizada;

4. Ano de publicação;

5. Veículo de publicação: livro, periódico científico, relatório, jornal diário, press release, matéria de revista, tese, dissertação ou carta;

6. Nome do veículo e/ou editor;

7. Trata: (a) da Agenda 2030 e seus ODS de forma geral; (b) da Agenda 2030 e seus ODS na América Latina; (c) de ODS específicos: 1, 2, $3 \ldots$

\section{Análise dos dados}

Foi realizada uma análise descritiva das publicações encontradas, incluindo: número de publicações por ano, veículos de publicação utilizados, assuntos tratados, instituições de afiliação dos autores e países onde se localizam. Os resultados são apresentados a seguir.

\section{Resultados}

\section{Assuntos abordados}

A descrição do objeto das publicações foi exaustiva, tendo sido registrado se a publicação trata da Agenda 2030 e seus ODS de forma geral, e/ou se trata da Agenda 2030 e seus ODS na América Latina e/ou se trata de ODS específicos - neste caso, registramos todos os ODS tratados por cada publicação. Uma publicação, assim, pode tratar de vários ODS concomitantemente.

O que se vê na figura 2 é que 26 publicações abordam os ODS de forma geral e 14 os abordam apenas quando referidos à América Latina. Dentre os ODS específicos, como esperado, o da saúde se destaca, sendo objeto de 127 das 278 publicações encontradas na busca. Noventa e nove das 127 publicações tratam exclusivamente do ODS 3 - Boa Saúde e Bem-estar, representando 34\% de todas as publicações identificadas neste trabalho. 


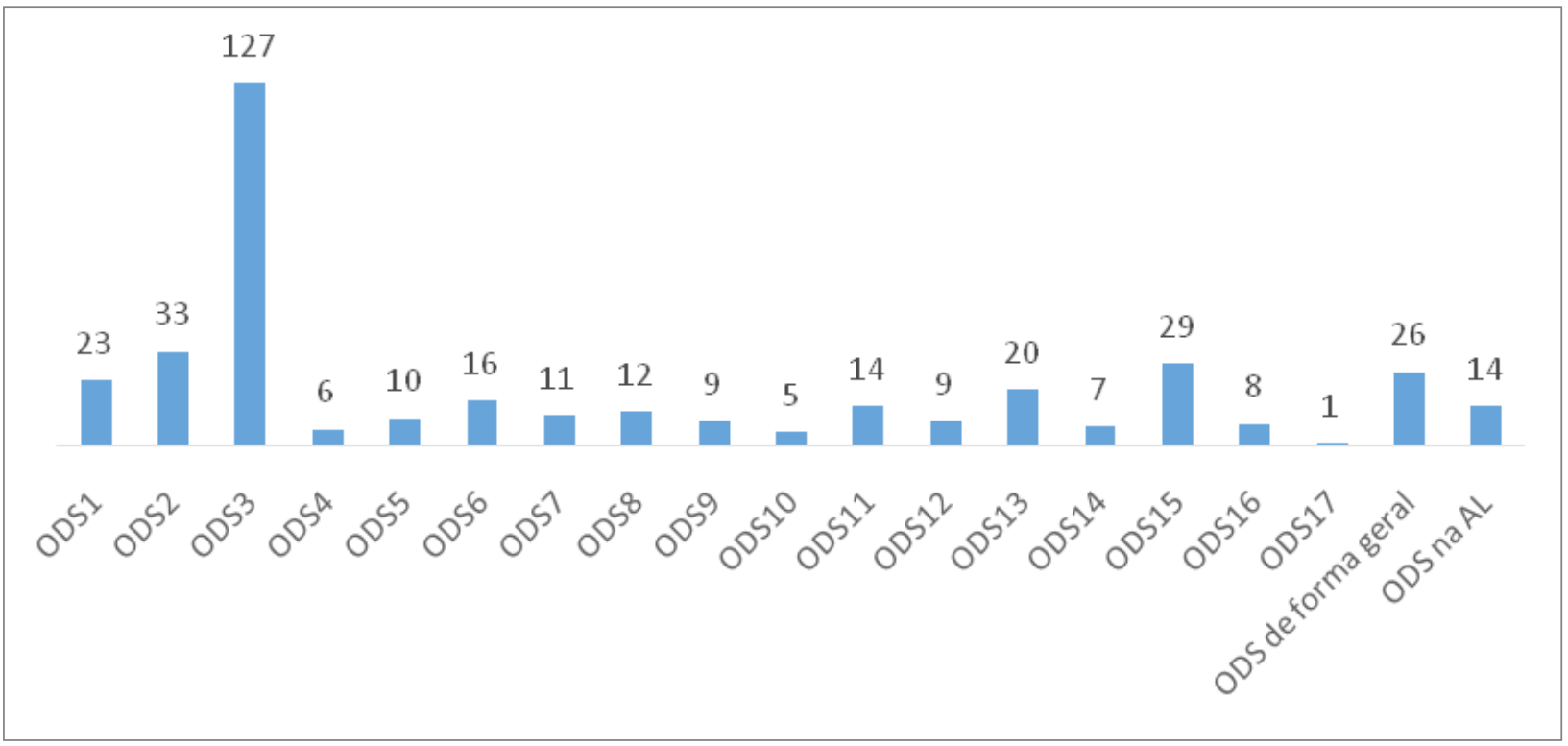

Figura 2 - Número de publicações por assunto $(n=278)$

Fonte: Os autores (2017).

\section{Número de publicações por ano sobre saúde}

A figura 3 mostra que o número de publicações sobre saúde cresceu no período analisado, chegando a 44 publicações em 2016. Já em 2017, até o mês de maio, contabilizavam-se apenas 25 publicações.

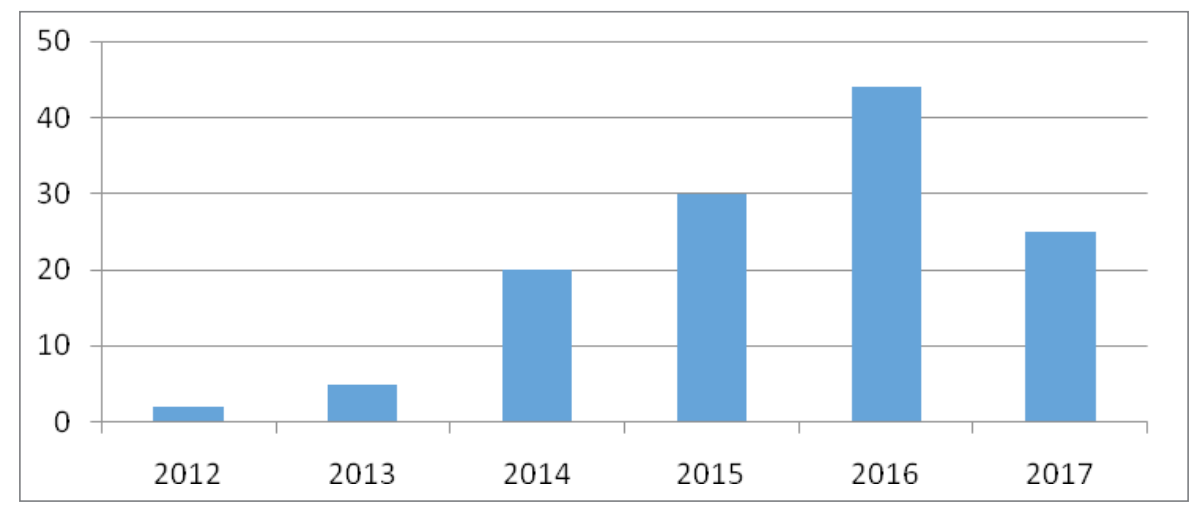

Figura 3 - Número de publicações por ano relativas ao ODS 3 - 2009/maio 2017 ( $n=127)$ Fonte: Os autores (2017).

\section{Veículos das publicações sobre saúde}

A grande maioria das publicações encontradas (92\%) é constituída de artigos científicos publicados em periódicos científicos. Também tiveram algum destaque os relatórios e as teses (2\%), enquanto cada um dos demais veículos representam 1\% do total encontrado, como mostra a figura 4 . 


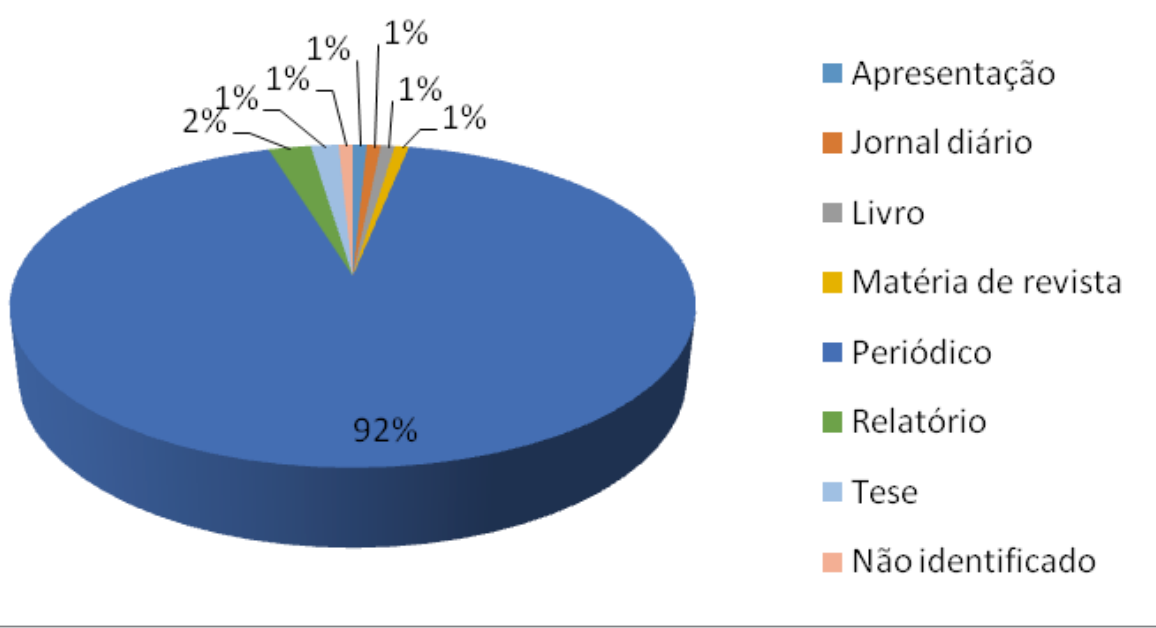

Figura 4 - Número de publicações sobre saúde por tipo de veículo $(n=127)$

Fonte: Os autores (2017).

\section{Primeiro autor das publicações sobre saúde}

É interessante notar que, em menos de um terço das publicações, o primeiro autor é latino-americano, como mostra a Figura 5 .

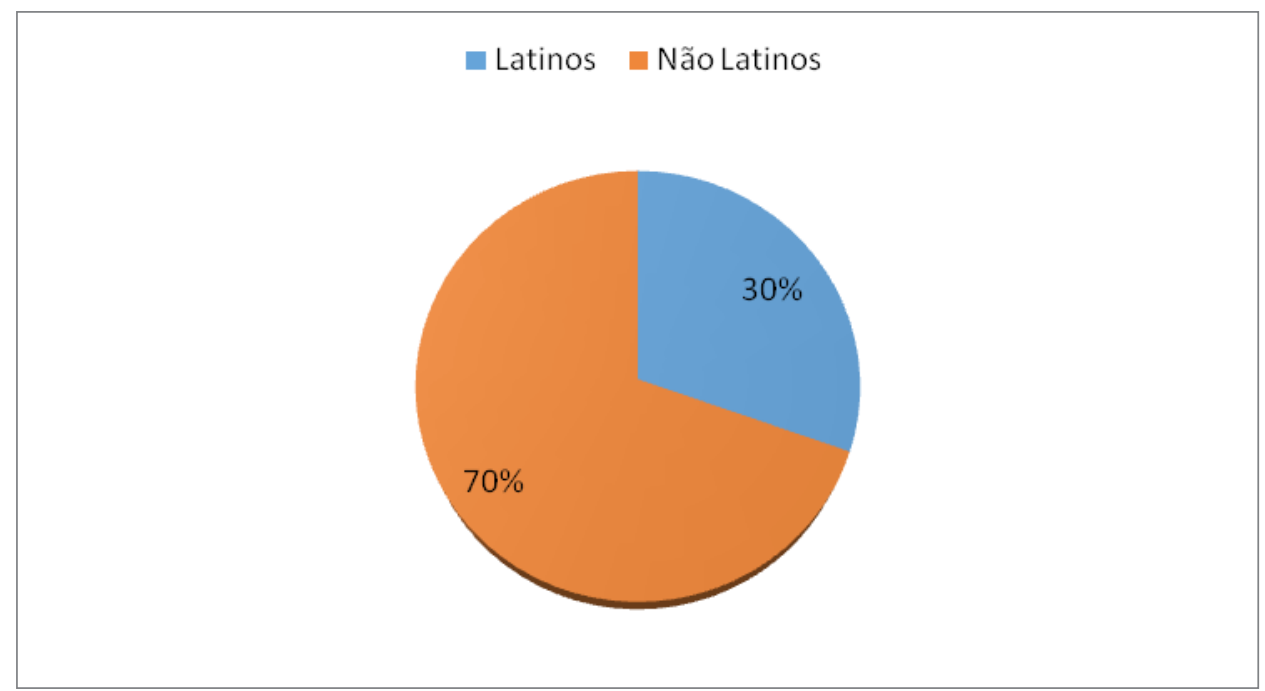

Figura 5 - Região de afiliação do primeiro autor $(n=126)$

Fonte: Os autores (2017).

Os Estados Unidos (EUA) foram o país que mostrou maior número de afiliações de primeiro autor, com 36 (29\% do total) das 126 publicações de autores identificados, seguido pelo Brasil com 29 publicações (23\%), Reino Unido e Suíça com nove (7\% cada), conforme a figura 6. Essa figura mostra os demais países de afiliação do primeiro autor das publicações, em ordem decrescente, registrando os 12 primeiros dos 27 países registrados. 


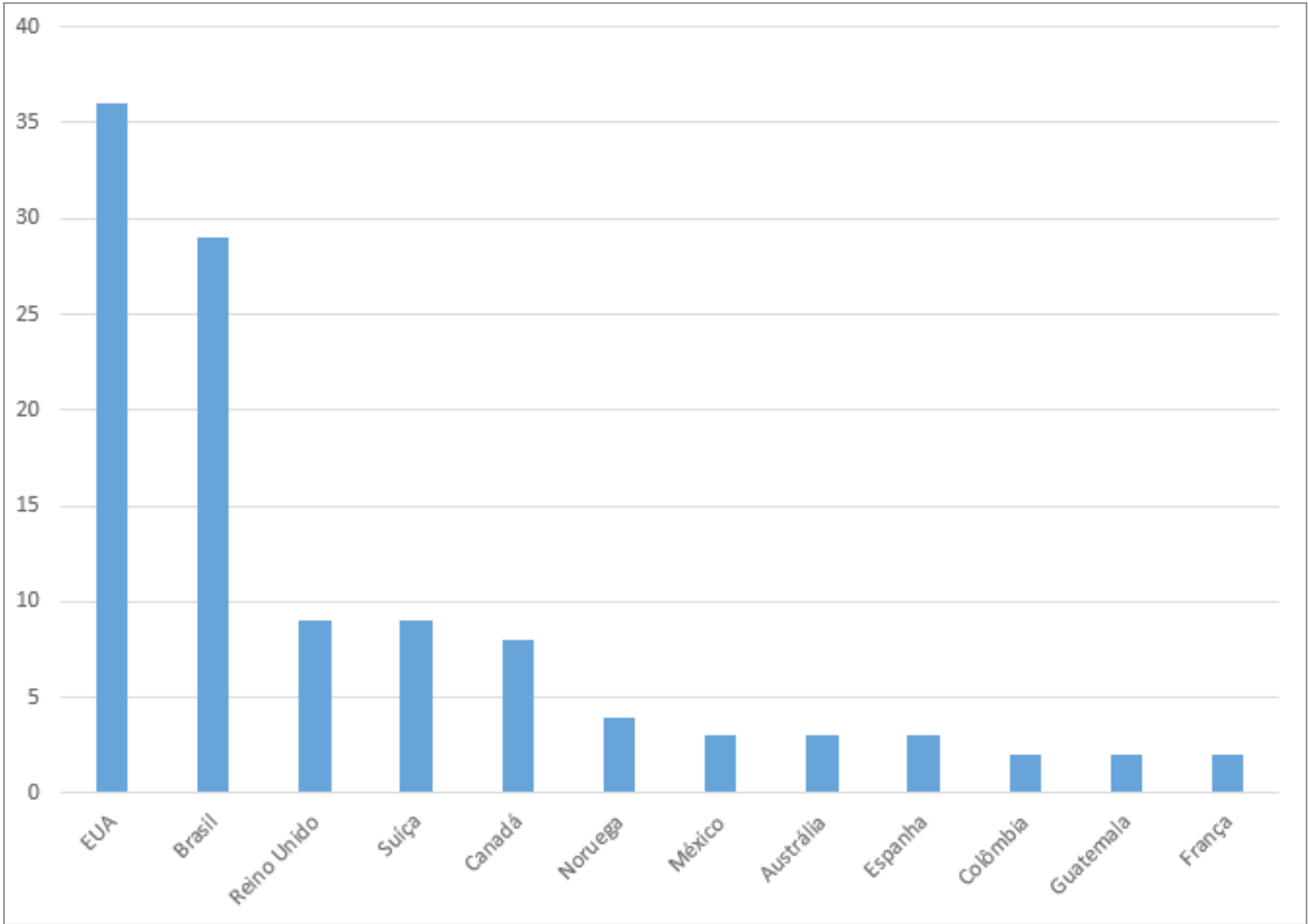

Figura 6 - País de afiliação do primeiro autor Fonte: Os autores (2017).

Considerando apenas os estudos que apresentavam como primeiro autor representantes da América Latina, verifica-se (Figura 6) que, em 76\% das publicações da região, eles vinculavam-se a uma organização brasileira, em $8 \%$ a uma organização mexicana, em $5 \%$ a uma colombiana ou guatemalteca e, em 3\%, argentina ou haitiana.

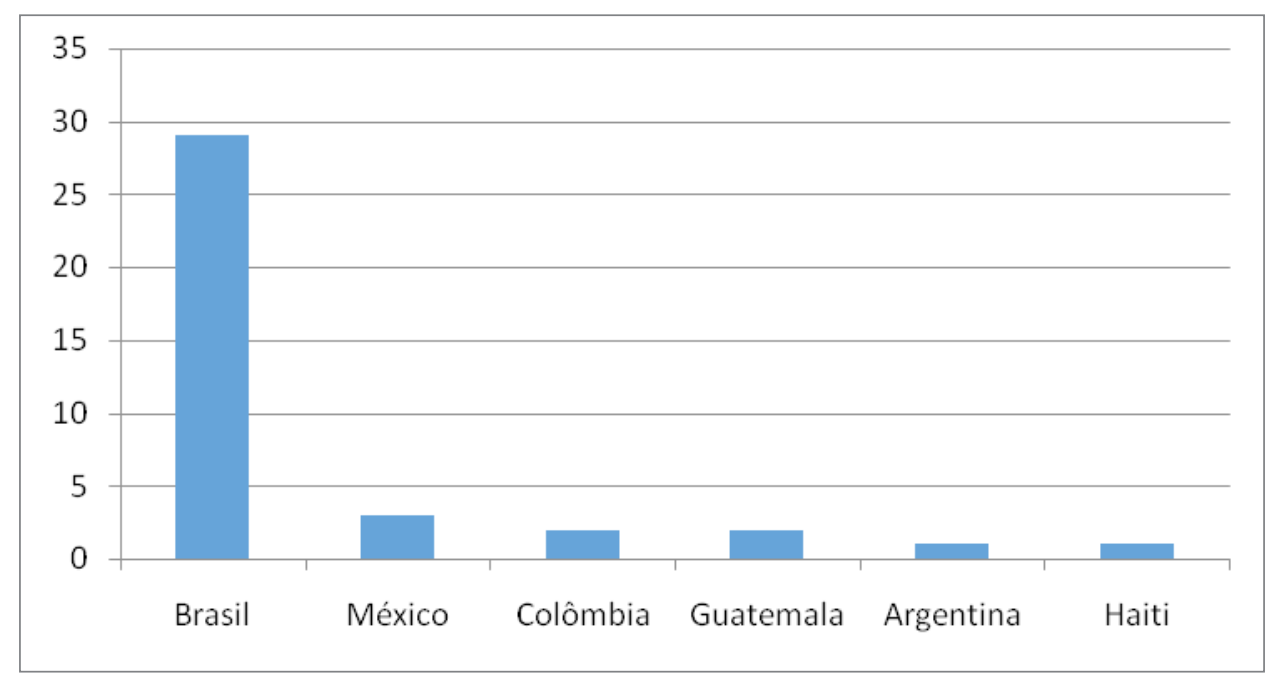

Figura 7 - País da América Latina de afiliação do primeiro autor Fonte: Os autores (2017). 
Cabe ressaltar que os seis países indicados na Figura 7 representam a totalidade das publicações relativas ao ODS 3 cujo primeiro autor é vinculado a uma organização latino-americana, listada no Quadro 1 a seguir.

Quadro 1-Organizações de afiliação do primeiro autor

\begin{tabular}{|c|c|c|}
\hline País & Organização & Publicações \\
\hline Argentina & CONICET - Consejo Nacional de Investigaciones Científicas y Técnicas & 1 \\
\hline \multirow{12}{*}{ Brasil } & Fiocruz - Fundação Oswaldo Cruz & 10 \\
\hline & UFPel - Universidade Federal de Pelotas & 5 \\
\hline & USP - Universidade de São Paulo & 3 \\
\hline & Hospital Público Regional Prefeito Osvaldo Rezende Franco & 2 \\
\hline & OPAS Brasil - Organização Pan-Americana da Saúde no Brasil & 2 \\
\hline & UFRJ - Universidade Federal do Rio de Janeiro & 1 \\
\hline & UFSC - Universidade Federal de Santa Catarina & 1 \\
\hline & Ministério da Saúde & 1 \\
\hline & Ulbra - Universidade Luterana do Brasil & 1 \\
\hline & UFC - Universidade Federal do Ceará & 1 \\
\hline & Ufes - Universidade Federal do Espírito Santo & 1 \\
\hline & Unipampa - Universidade Federal do Pampa & 1 \\
\hline \multirow{2}{*}{ Colômbia } & Universidad Nacional de Colombia & 1 \\
\hline & Universitaria de Ciencias de la Salud & 1 \\
\hline Guatemala & CEGSS - Centro de Estudios para la Equidad y Gobernanza en los Sistemas de Salud & 2 \\
\hline Haiti & Haiti Philantropy Inc. & 1 \\
\hline \multirow{2}{*}{ México } & Fundación Mexicana para la Salud & 1 \\
\hline & Universidad de Sonora & 2 \\
\hline
\end{tabular}

Fonte: Os autores (2017).

\section{O conjunto de autores das publicações sobre saúde}

Quando é considerada a totalidade dos autores envolvidos com as publicações registradas, a situação se inverte e mais da metade das publicações passam a possuir pelo menos um de seus autores vinculado às instituições latino-americanas, conforme mostra a Figura 8. Mas é interessante reparar que $47 \%$ das publicações sobre o ODS 3 na América Latina foram escritas apenas por autores não latinos. 


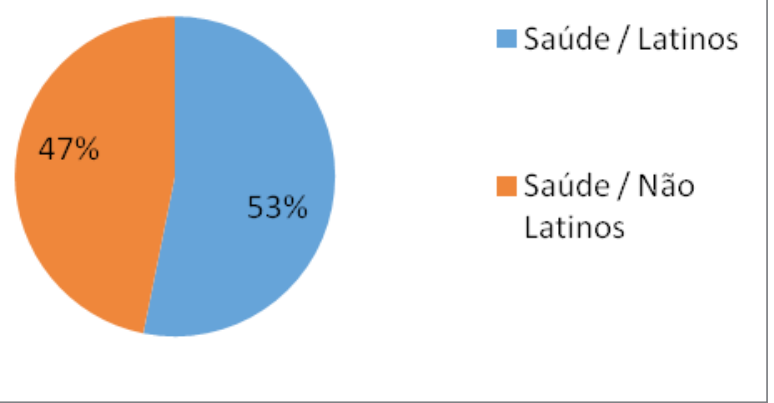

Figura 8 - Publicações sobre saúde que possuem ao menos um autor vinculado à organização latino-americana $(n=126)$ Fonte: Os autores (2017).

\section{Instituições de vinculação do conjunto de autores sobre saúde}

O universo aqui descrito é o conjunto de autores das publicações identificadas. Um mesmo autor pode aparecer em diversas publicações, e a instituição à qual está vinculado será contabilizada diversas vezes. Uma publicação pode ter vários autores de uma mesma instituição - nesse caso também a instituição será contabilizada várias vezes. Tem-se, assim, um indicativo do tamanho de equipe e produção.

A Figura 8 representa o número de vezes que os países sedes das instituições de afiliação dos autores aparecem nas publicações levantadas. Assim como se observou para o primeiro autor, os Estados Unidos são o país da maioria dos autores, ficando o Brasil em segundo lugar, Suíça em terceiro e Reino Unido em quarto. Por questões gráficas, a figura mostra apenas os 11 primeiros dos 43 países de afiliação dos autores das publicações.

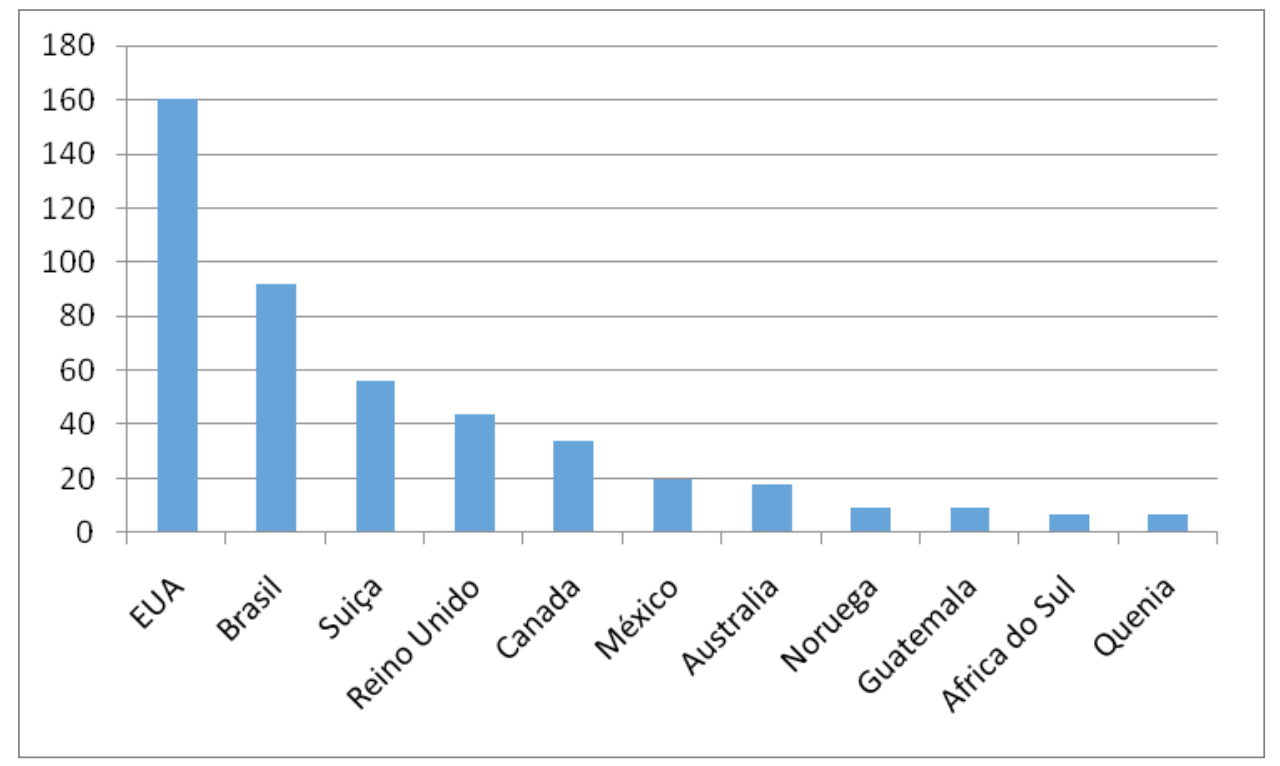

Figura 8 - Número de vezes que as instituições do país aparecem na autoria das publicações Fonte: Os autores (2017).

O Brasil se destaca no cenário latino-americano, como se vê na Tabela 1. O número de vezes que autores vinculados às instituições brasileiras publicaram é maior que a soma de todos os demais países da região. 


\begin{tabular}{lcc}
\hline País & $\begin{array}{c}\text { No de vezes que instituições do } \\
\text { país aparecem no campo autoria }\end{array}$ & \% \\
\hline Brasil & 92 & 64 \\
México & 20 & 14 \\
Guatemala & 9 & 6 \\
Chile & 5 & 3 \\
Peru & 5 & 3 \\
Colômbia & 4 & 3 \\
Nicarágua & 4 & 3 \\
Argentina & 2 & 1 \\
El Salvador & 1 & 1 \\
Guiana & 1 & 1 \\
Haiti & 1 & 1 \\
Total & $\mathbf{1 4 4}$ & $\mathbf{1 0 0}$ \\
\hline
\end{tabular}

Fonte: Os autores (2017).

O Quadro 2 apresenta as instituições do conjunto de autores latino-americanos das 126 publicações sobre saúde. É possível observar que o Brasil foi o país com o maior número de organizações que publicaram sobre o ODS 3 - Saúde e Bem-Estar, com 17 instituições no total.

O número de instituições por país guarda relação direta com o número de vezes que as instituições do país aparecem na autoria das publicações (Figura 8). Assim, o país com maior número de organizações a publicar sobre o tema, depois do Brasil, foi o México (com 10 organizações), seguido da Colômbia (com quatro) e do Peru (com três).

A grande maioria das instituições latino-americanas identificadas é constituída de universidades - como era de se esperar, pois as publicações são majoritariamente acadêmicas. A Fiocruz se destaca, aparecendo 37 vezes na autoria dos trabalhos, seguida pela Universidade Federal de Pelotas, no Brasil, e pela Universidade de Sonora, no México (nove vezes).

Deve-se destacar a presença de organizações internacionais que publicaram sobre o tema na região, como OPAS Brasil e El Salvador, e Unicef (United Nations Children's Fund) México e Peru. 
Quadro 2 - Organizações de afiliação dos autores

\begin{tabular}{|c|c|c|}
\hline País & Organização & Publicações \\
\hline \multirow{2}{*}{ Argentina } & CONICET - Consejo Nacional de Investigaciones Científicas y Técnicas & 1 \\
\hline & Instituto de Efectividad Clínica Sanitaria & 1 \\
\hline \multirow{18}{*}{ Brasil } & Fiocruz - Fundação Oswaldo Cruz & 37 \\
\hline & UFPel - Universidade Federal de Pelotas & 9 \\
\hline & Ministério da Saúde & 6 \\
\hline & OPAS Brasil - Organização Pan-Americana da Saúde no Brasil & 5 \\
\hline & UFSC - Universidade Federal de Santa Catarina & 5 \\
\hline & USP - Universidade de São Paulo & 4 \\
\hline & UnB - Universidade de Brasília & 3 \\
\hline & UFRJ - Universidade Federal do Rio de Janeiro & 3 \\
\hline & Ulbra - Universidade Luterana do Brasil & 3 \\
\hline & Hospital Público Regional Prefeito Osvaldo Rezende Franco & 2 \\
\hline & Unicamp - Universidade Estadual de Campinas & 2 \\
\hline & UFC - Universidade Federal do Ceará & 2 \\
\hline & Universidade Federal de Santa Maria & 1 \\
\hline & Universidade Federal de Lavras & 1 \\
\hline & Ufes - Universidade Federal do Espírito Santo & 1 \\
\hline & Instituto Butantan & 1 \\
\hline & UFBA - Universidade Federal da Bahia & 1 \\
\hline & Unipampa - Universidade Federal do Pampa & 1 \\
\hline \multirow{2}{*}{ Chile } & Universidad de Chile & 4 \\
\hline & Fonasa - Fondo Nacional de Salud & 1 \\
\hline \multirow{4}{*}{ Colômbia } & Universidad Nacional de Colombia & 1 \\
\hline & Universitaria de Ciencias de la Salud & 1 \\
\hline & Universidad Ciencias Aplicadas y Ambientales & 1 \\
\hline & Pontificia Universidad Javeriana & 1 \\
\hline El Salvador & OPAS - Organización Panamericana de la Salud & 1 \\
\hline Guatemala & CEGSS - Centro de Estudios para la Equidad y Gobernanza en los Sistemas de Salud & 8 \\
\hline Guiana & O'Neil Institute for National and Global Health & 1 \\
\hline Haiti & Haiti Philantropy Inc. & 1 \\
\hline \multirow{10}{*}{ México } & Universidad de Sonora & 9 \\
\hline & Instituto Nacional de Salud Publica de México & 4 \\
\hline & Hospital Infantil de México Frederico Gómez & 1 \\
\hline & Fundación Mexicana para la Salud & 1 \\
\hline & UNAM - Universidad Nacional de México & 1 \\
\hline & Instituto Mexicano de Oftalmologia & 1 \\
\hline & Um Kilo de Ayuda & 1 \\
\hline & Unicef & 2 \\
\hline & Instituto Centro-Americano de Salud & 1 \\
\hline & Consultor independente & 1 \\
\hline \multirow{3}{*}{ Peru } & Universidad Peruana Cayetano Heredia & 2 \\
\hline & Salud sin Limites & 2 \\
\hline & Unicef - United Nations Children's Fund & 1 \\
\hline
\end{tabular}

Fonte: Os autores (2017). 


\section{Discussão e conclusões}

Este trabalho consistiu no levantamento quantitativo das publicações sobre a Agenda 2030 e seus ODS na América Latina, ou de autores afiliados a instituições da região, com foco em saúde, por meio de pesquisa bibliográfica nos mais importantes bancos de dados nacionais e internacionais. Ele faz parte de uma ampla investigação que também trata do conteúdo dos estudos e do seu escopo, que não foi objeto da reflexão que deu origem a este artigo.

Ao utilizar as bases de dados indexadas como fonte de informação, já se previa que essa busca seria enriquecida com publicações acadêmicas, uma vez que thinktanks e organizações voltadas para a política têm objetivo mais prático e tendem a divulgar seus resultados em meios de comunicação de massa, revistas de órgãos governamentais ou classes profissionais. Efetivamente, o que se observou foi que: artigos científicos foram a grande maioria dos documentos recuperados, e as universidades representaram a maior parte das instituições identificadas.

No entanto, a revisão da bibliografia trouxe resultados interessantes. Praticamente a metade das publicações sobre saúde no âmbito da Agenda 2030 na América Latina (47\%) é feita sem a participação de qualquer organização latino-americana. Em apenas 30\% dos casos, o primeiro autor pertence a uma das organizações que se enquadram nesse perfil. Este dado revela uma realidade acadêmica importante: a Agenda 2030 e seus Objetivos de Desenvolvimento Sustentável ainda estão ausentes nas produções científicas de grande parte da América Latina.

Isso se torna ainda mais relevante quando se observa a extrema concentração da produção latinoamericana. O Brasil se destaca, tanto em número de instituições, como na frequência com que estas publicam. No Brasil, a Fiocruz se destaca, liderando a produção acadêmica sobre a Agenda 2030 na área da saúde.

Este trabalho, entretanto, apresenta uma limitação temporal - o levantamento foi encerrado em maio de 2017, quando, efetivamente se observava uma tendência de aumento no número de publicações. Sua atualização fica como forte sugestão para trabalhos futuros.

\section{Referências}

1. Naciones Unidas. Transformar nuestro mundo: la Agenda 2030 para el Desarrollo Sostenible [Internet]. A/Res/70/1. Resolución de la Asamblea General de las Naciones Unidas, 21 de octubre de 2015 [acceso en 2018 fev 2]. Disponible en: http://www.un.org/ga/search/view doc.asp?symbol=A/ $\underline{\mathrm{RES}} / 70 / 1$ \&referer=/english/\&Lang=S.

2. Nações Unidas Brasil. Transformando nosso mundo: a Agenda 2030 para o Desenvolvimento Sustentável [Internet]. 2015 [citado 2018 fev. 2]. Disponível em: https://nacoesunidas.org/pos2015/agenda2030.

3. Buss P, Quental C, Minayo M, Tobar S, Galvão LA, organizadores. Implementación de La Agenda 2030 y SUS ODS a nivel nacional en América Latina: respuestas institucionales en 7 países de la región. Rio de Janeiro: Fundação Oswaldo Cruz; 2018.

4. McGann JG. 2017 Global Go To Think Tank Index Report [Internet]. University of Pennsylvania; 2017 [citado em 2018 fev 2]. TTCSP Global Go To Think Tank Index Reports 13. Disponível em: https:// repository.upenn.edu/think tanks/13.

5. Galvão LAG. Saúde Pública na era do desenvolvimento sustentável: análise de sua evolução e evidências [tese]. Rio de Janeiro; Universidade Federal do Rio de Janeiro; 2015. 170 f. 